\title{
High-Fidelity Prediction of Megapixel Longitudinal Phase-Space Images of Electron Beams Using Encoder-Decoder Neural Networks
}

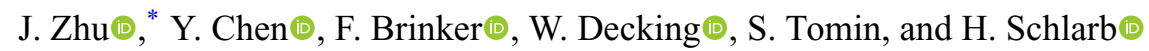 \\ Deutsches Elektronen-Synchrotron DESY, Notkestrasse 85, Hamburg 22607, Germany
}

(Received 22 February 2021; revised 21 June 2021; accepted 15 July 2021; published 3 August 2021)

\begin{abstract}
Modeling of large-scale research facilities is extremely challenging due to complex physical processes and engineering problems. Here, we adopt a data-driven approach to model the longitudinal phase-spacediagnostic beamline at the photoinector of the European XFEL with an encoder-decoder neural-network model. A deep convolutional neural network (decoder) is used to build images, measured on the screen, from a small feature map generated by another neural network (encoder). We demonstrate that the model, trained only with experimental data, can make high-fidelity predictions of megapixel images for the longitudinal phase-space measurement without any prior knowledge of photoinjectors or electron beams. The prediction significantly outperforms existing methods. We also show the scalability and interpretability of the model by sharing the same decoder with more than one encoder, used for different setups of the photoinjector, and propose a pragmatic way to model a facility with various diagnostics and working points. This opens the door to a way of accurately modeling a photoinjector using neural networks and experimental data. The approach can possibly be extended to the whole accelerator and even other types of scientific facility.
\end{abstract}

DOI: 10.1103/PhysRevApplied.16.024005

\section{INTRODUCTION}

The operation of large-scale scientific user facilities such as the European XFEL [1] is very challenging, as it is necessary to meet the specifications of various user experiments [2] and to be capable of switching the machine status rapidly. Machine learning, especially deep learning, has provided powerful tools for accelerator physicists to build fast-prediction surrogate models [3-5] and to extract essential information [6-8] from large amounts of data in recent years. These machine-learning models can be extremely useful for building virtual accelerators, which are capable of making fast predictions of the behavior of beams [9], assisting accelerator tuning by virtually bringing destructive diagnostics online [4], providing an initial guess of input parameters for model-independent adaptive feedback control algorithms [10,11], and driving modelbased feedback control algorithms [12]. Deep learning is a subfield of machine learning based on artificial neural networks [13]. One way of training a neural-network model is to make use of simulated data. However, beamdynamics simulations are typically carried out under different theoretical assumptions about collective effects such as space-charge forces, wakefields, and coherent synchrotron radiation. In addition, electron emission from a photocathode is governed by multiple physical processes and is even

*jun.zhu@desy.de more difficult to simulate [14]. Moreover, aging of accelerator components affects the long-term operation of a facility but is generally not included in simulations. As a result, it is extremely challenging to achieve good agreement between simulations and measurements for a large range of machine operation parameters, even by exploiting complicated physical models [15]. Furthermore, it can be prohibitively expensive to collect a large amount of high-resolution simulation data [16].

Previous work has demonstrated prediction of the measured longitudinal phase space at the exit of the LCLS accelerator using the L1S phase and a shallow multilayer perceptron [4]. The images were cropped to $100 \times 100$ pixels, and the phase-space distribution needed to be centered in order to produce reasonable results. Nonetheless, the predicted longitudinal phase space was blurry and had significant artifacts in the background. Moreover, the current profile was predicted by using another multilayer perceptron instead of being extracted directly from the predicted longitudinal phase space. Indeed, a multilayer perceptron consisting of purely fully connected layers has intrinsic limitations in image-related tasks, as it is intended to find the connections between each pair of nodes between each two adjacent layers. First of all, this unnecessarily complicates the training of the neural network, as the pixels representing the phase-space distribution apparently have little connection with the majority of the background pixels. Secondly, the number of parameters scales at least 
proportionally to the number of pixels in the image, which makes it impractical to apply this technique to megapixel images due to the huge memory requirement. In Ref. [17], convolutional and upsampling layers were used in predicting simulated longitudinal phase spaces for the LCLS. The results do not show artifacts in the background. However, details of the study were not reported.

In this paper, we propose an encoder-decoder neuralnetwork model to make high-fidelity predictions of megapixel images measured on a screen, and present an experimental demonstration of it at the longitudinal phasespace-diagnostic beamline at the injector of the European XFEL. This method can provide not only high-quality virtual diagnostics while online tuning of a photoinjector is being performed, but also very fast machine-specific offline simulation. Besides the performance, another major advantage of this approach over existing ones $[4,12]$ is that the output of our model is the full image from the camera. Therefore, the same neural-network structure can be applied to distributions with different footprints, for example, when beam properties such as the energy spread and bunch length change significantly. The physical properties concerned can then be extracted by using well-established routines. The model learns only from the experimental data, without any prior knowledge of rf photoinjectors or electron beams, which makes it potentially applicable to many other image-based diagnostics. More importantly, we demonstrate the scalability and interpretability of the model by sharing the same decoder with encoders used for different setups of the photoinjector, and propose a pragmatic way to model a photoinjector with various diagnostics and working points. It must be pointed out that our method is essentially different from the variational autoencoder [18] and the generative adversarial network [19], both of which learn a joint probability distribution from the training dataset, allowing it to synthesize images from random noise. In this study, however, we aim to find an explicit mapping between the input parameters and the output image.

\section{DEEP-LEARNING MODEL}

\section{A. Neural network}

The general architecture of the encoder-decoder model is illustrated in Fig. 1(a). More generally, given an input $\mathbf{x} \in \mathbb{R}^{m}$ and a measurement $\mathbf{y} \in \mathbb{R}^{n}$, the model is asked to learn two neural networks $g_{\varphi}: \mathbb{R}^{m} \rightarrow \mathbb{R}^{c}$ and $f_{\theta}: \mathbb{R}^{c} \rightarrow$ $\mathbb{R}^{n}$, where $\mathbb{R}^{c}$ is the latent space and $\mathbf{z} \in \mathbb{R}^{c}$ is called the latent features. Both $m$ and $n$ can be very large, as modern area detectors typically have millions of pixels. The learning process can be described as minimizing a loss function $\mathcal{L}\left(\mathbf{y}, f_{\theta}\left(g_{\varphi}(\mathbf{x})\right)\right)$ using a gradient descent algorithm. Therefore, the model learns only from nonfixed input data $\tilde{\mathbf{x}}$, and the encoder can be simplified to $g_{\varphi}(\mathbf{x})=g_{\varphi}(\tilde{\mathbf{x}} \mid \overline{\mathbf{x}})=g_{\varphi}(\tilde{\mathbf{x}})$, where $\overline{\mathbf{x}}$ is the fixed input data and $\overline{\mathbf{x}} \oplus \tilde{\mathbf{x}}=\mathbf{x}$. Here we assume that the influence of the jitter of $\overline{\mathbf{x}}$ is negligible. (a)

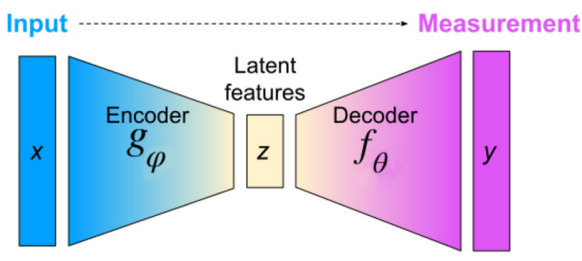

(c)

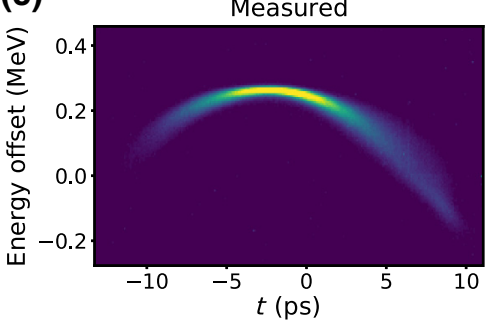

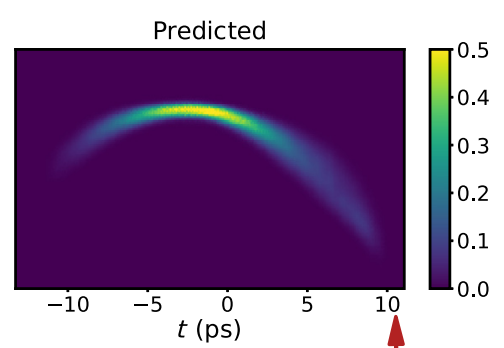

(b)

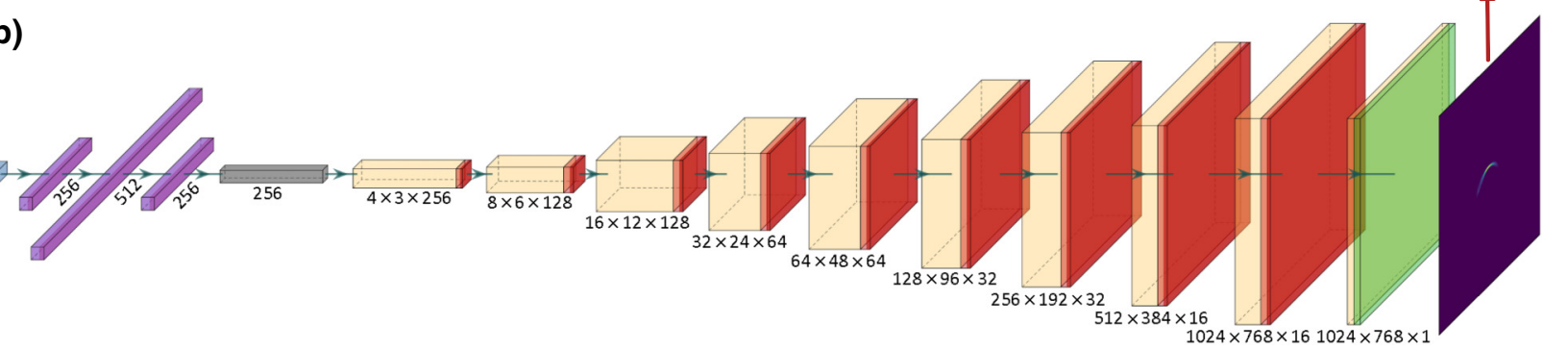

FIG. 1. (a) General architecture of the encoder-decoder model. (b) Diagram of the neural network. The leftmost (blue) box represents the input layer. It is followed by three fully connected layers (the encoder), shown in purple, with each layer activated by a leaky rectified-linear-unit (ReLU) function. The latent space is depicted in gray. The ten yellow boxes represent the transposed convolutional layers (the decoder). Each transposed convolutional layer is followed by a batch normalization layer [13] and is activated by the leaky ReLU function except for the last one, which is activated by a sigmoid function, depicted in green. The kernel sizes of the first and second transposed convolutional layers are $3 \times 4$ and $3 \times 3$, respectively, and the kernel sizes of the other eight transposed convolutional layers are all $5 \times 5$. The total number of trainable parameters is 1898161 . (c) Example of longitudinal phase spaces cropped from the measured image, and the corresponding prediction. 
Although it can be challenging for neural networks to learn a universal approximator for the whole input parameter space of an accelerator, this approach can be well suited for user facilities, as they are typically operated at a finite number of working points.

The detailed structure of the model is shown in Fig. 1(b). We use a multilayer perceptron to learn latent features and then map them to the image on the screen using a concatenation of transposed convolutional layers [20]. The transposed convolutional layer performs the transformation in the direction opposite to a normal convolution, which projects localized feature maps to a higher-dimensional space. Despite the deepness of the neural network, a single prediction takes only about $20 \mathrm{~ms}$ on an NVIDIA Tesla P100-16GB graphics card, which is orders of magnitude faster than a standard beam-dynamics simulation.

\section{B. Loss function}

Neural networks are trained using the minibatch stochastic gradient decent optimization algorithm [13] driven by a loss function. For most regression problems, the choice of the loss function defaults to the mean squared error (MSE) [4-6,17]. However, a MSE loss function treats pixels as uncorrelated features and is found to result in overly smoothed images and loss of high-frequency features in high-resolution image-generation applications [21]. In our model, the loss function takes into account the correlations between adjacent pixels and is given by

$$
L_{\text {batch }}=\frac{1}{N_{b}} \sum_{i=1}^{N_{b}}\left[1-h\left(\mathbf{y}_{i}, \hat{\mathbf{y}}_{i}\right)\right],
$$

where $N_{b}$ is the batch size for training, $\mathbf{y}$ is the measurement, $\hat{\mathbf{y}}$ is the prediction, and $h$ is the structural similarity index measure (SSIM) [22] for multiple scales, written as

$$
\begin{aligned}
h(\mathbf{y}, \hat{\mathbf{y}})= & {\left[\frac{1}{N_{p}^{(M)}} \sum_{\substack{\forall \mathbf{p} \in \mathbf{y}^{(M)} \\
\forall \hat{\mathbf{p}} \in \hat{\mathbf{y}}^{(M)}}} l(\mathbf{p}, \hat{\mathbf{p}}) c(\mathbf{p}, \hat{\mathbf{p}}) s(\mathbf{p}, \hat{\mathbf{p}})\right]^{\alpha_{M}} \prod_{j=0}^{M-1} } \\
& \times\left[\frac{1}{N_{p}^{(j)}} \sum_{\substack{\forall \mathbf{p} \in \mathbf{y}^{(j)} \\
\forall \hat{\mathbf{p}} \in \hat{\mathbf{y}}^{(j)}}} c(\mathbf{p}, \hat{\mathbf{p}}) s(\mathbf{p}, \hat{\mathbf{p}})\right]^{\alpha_{j}}
\end{aligned}
$$

Here, $l(\mathbf{p}, \hat{\mathbf{p}}), c(\mathbf{p}, \hat{\mathbf{p}})$, and $s(\mathbf{p}, \hat{\mathbf{p}})$ measure the distortions in luminance, contrast, and structure [22], respectively, between a uniform sliding window $\mathbf{p}$ of size $8 \times 8$ pixels on the measured image $\mathbf{y}^{(j)}$ and its counterpart $\hat{\mathbf{p}}$ on the predicted image $\hat{\mathbf{y}}^{(j)}$. The number of pixels in $\mathbf{y}^{(j)}$ is denoted as $N_{p}^{(j)}$. The superscript $j \in\{0, \ldots, M\}$ indicates that the image is downsampled by a factor of $2^{j}$ using

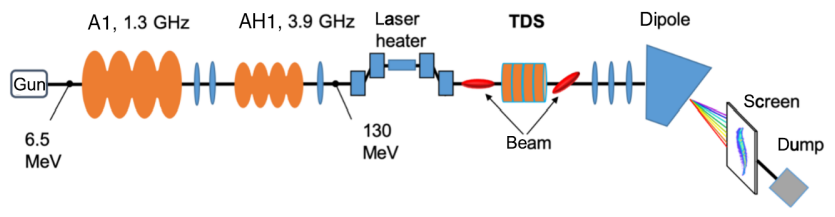

FIG. 2. Schematic illustration of the European XFEL photoinjector and its diagnostic beamline. The phases of the gun, the 1.3 $\mathrm{GHz}$ cryomodule (A1), and the $3.9 \mathrm{GHz}$ cryomodule (AH1) are used as input to predict the image on the screen. The laser heater is switched off during the experiment.

average pooling. Because $l(\mathbf{p}, \hat{\mathbf{p}}), c(\mathbf{p}, \hat{\mathbf{p}})$, and $s(\mathbf{p}, \hat{\mathbf{p}})$ all range between 0 and 1 for nonnegative image data, having $\alpha_{j}<1$ prevents the model from overfitting on fine local features that could be induced by machine jitter. Comparisons between images at different scales obviously enable the model to learn the correlations between pixels over a wider area. We empirically choose $M=2$ with $\alpha_{0}=0.05$, $\alpha_{1}=0.30$, and $\alpha_{2}=0.65$ for this study.

\section{EXPERIMENTAL RESULTS}

\section{A. Experimental setup}

The experiment is carried out at the injector of the European XFEL [23], and the layout of the beamline is shown in Fig. 2. The nominal beam energy is approximately $130 \mathrm{MeV}$, which is measured at the maximummean-momentum-gain (MMMG) phases of the gun and A1 and at the zero-crossing [24] phase of AH1. We refer to this working point as the reference working point and the corresponding phases as the reference phases. The bunch charge is around $250 \mathrm{pC}$. The transverse deflecting structure (TDS) and the dipole magnet are used to measure the longitudinal phase space at a resolution of about 0.047 ps/pixel and $0.0031 \mathrm{MeV} /$ pixel. We collect data for two different working points (WPs). For WP1, the phases of the gun, A1, and AH1 are uniformly sampled within $\pm 3^{\circ}$, $\pm 6^{\circ}$, and $\pm 6^{\circ}$ relative to the respective reference phases. It is worth mentioning that the actual MMMG phase of A1 and the zero-crossing phase of AH1 shift as the gun phase varies, due to the change in time of flight. For WP2, AH1 is switched off, and the gradient of Al is reduced accordingly to keep the nominal beam energy at approximately $130 \mathrm{MeV}$. The sample ranges of the gun and A1 phases remain the same. The inputs for WP1 and WP2 are summarized in Table I.

\section{B. Data analysis}

The original image size is $1750 \times 2330$ pixels. After background subtraction and normalization, all pixel values below 0.01 are set to 0 . In order to have a reasonable training time during our study with limited computational resources, all the images are slightly cropped at the same locations and then downsampled to $768 \times 1024$ 
TABLE I. Input parameters and their ranges for the two working points. The lengths of $\tilde{\mathbf{x}}$ for WP1 and WP2 are 3 and 2 , respectively.

\begin{tabular}{lcc}
\hline \hline & WP1 & WP2 \\
\hline Gun phase (deg) & -3 to 3 & -3 to 3 \\
A1 phase (deg) & -6 to 6 & -6 to 6 \\
AH1 phase (deg) & -6 to 6 & - \\
\hline \hline
\end{tabular}

pixels. The model is implemented and trained using the machine-learning framework TensorFlow [25], version 2.3.1. For training, we adopt the weight initialization used in Ref. [26] and the Adam optimizer [27]. In total, 3000 shots are collected for each working point. $80 \%$ of the data are used for training, and the rest are used for testing. The minibatch size is 32 during training. The single-scale $\operatorname{SSIM}\left(M=0\right.$ and $\left.\alpha_{0}=1.0\right)$ is used as a metric to evaluate the performance of the trained model. It must be pointed out that this metric cannot be used an absolute quantitative measurement, because low metric values can be induced for various reasons such as a shift between the predicted and the measured longitudinal phase spaces, a blurry prediction, and mismatched backgrounds. As mentioned previously, the proposed model does not require the phase-space distribution to be centered. Figures 3(a) and 3 (b) show the distributions of the $x$ and $y$ coordinates, respectively, of the centers of mass for the preprocessed images. Evidently, the centers of mass are distributed over a wide area of $160 \times 46$ pixels for WP1 and $122 \times 52$ pixels for WP2.

In machine learning, it is crucial that information about the test dataset should not leak into the training dataset, in order to avoid overfitting of the model. Therefore, the data points in the test dataset should not appear again in the training dataset. Figure 3(c) shows that there are no duplicated phase vectors in the data, for both WP1 and WP2. Therefore, randomly split training and test datasets will not contain the same data point.

The model for WP1 is trained with a learning rate of $10^{-3}$ for 200 epochs and then $3 \times 10^{-4}$ for 400 epochs. In total, the process takes approximately $10 \mathrm{~h}$. The performance of the model over the test dataset is as high as $0.9955 \pm 0.00202$. An example predicted image is shown in Fig. 4(a). The model successfully predicts the electron distribution recorded on the screen, with a clean background. The predicted longitudinal phase space shown in Fig. 4(c) and the measured longitudinal phase space shown in Fig. 4(b) agree very well at different longitudinal positions in the bunch, which experience different nonlinear processes during emission from the cathode and during travel through the beamline. We also train another model to demonstrate the influence of the loss function. The second model has the same structure as the first but uses the MSE as the loss function. The phase space shown in
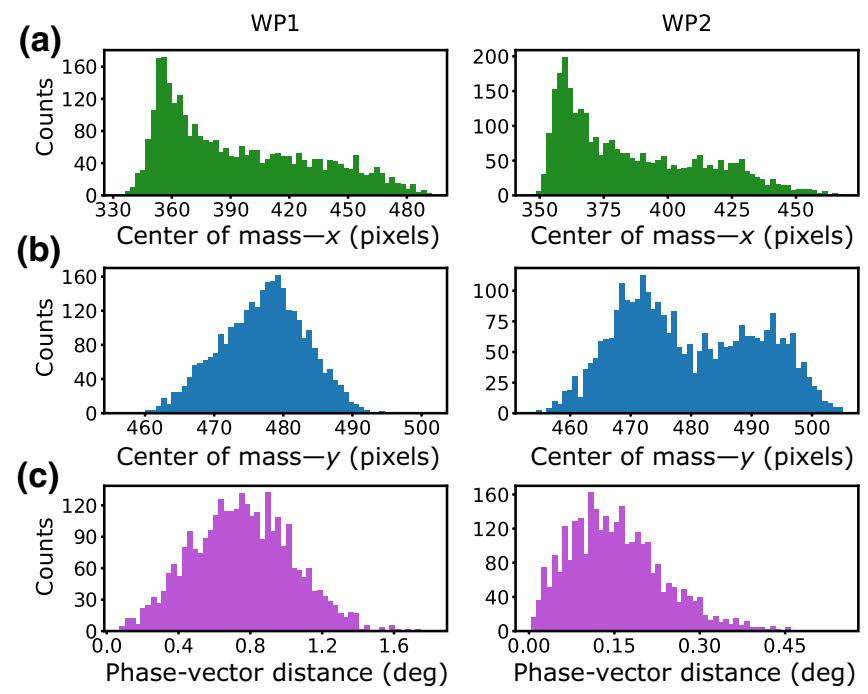

FIG. 3. Statistics of the data for WP1 (first column) and WP2 (second column). (a),(b) Histograms of the $x$ and $y$ coordinates of the centers of mass for the preprocessed images. (c) Histogram of the minimum Euclidean distance between the input phase vector of each data point and those of the remaining points. The presence of no counts in the zero bin indicates that no data points are shared between the training and test datasets.

Fig. 4(d) is apparently blurrier than that shown in Fig. 4(c), although the performance of the model over the test dataset is $0.9948 \pm 0.00187$. Figures $4(\mathrm{e})-4(\mathrm{~g})$ further compare the current profiles, the energy spectra, and the rms slice energy spreads of the longitudinal phase spaces shown in Figs. 4(b)-4(d). The predictions all agree excellently with the measurements except for the slice energy spread along the first half of the bunch. This can be observed from the sharpness of the image in the corresponding regions. This is understandable because the input does not cover the complete state of the photoinjector. For example, the arrival-time jitter of the photocathode laser [28] has a nonnegligible impact on these regions, which possess only a few pixels.

The ability to measure high peak currents is of critical importance for a free-electron laser facility. Although all the current profiles resemble each other in this study, the energy spectra vary dramatically during the phase scan. Figures 5(a) and 5(b) show two typical results with high peaks in the energy spectra. Another model is trained on WP2 data. This model is trained with a learning rate of $10^{-3}$ for 200 epochs and then $3 \times 10^{-4}$ for 100 epochs. The performance of the model over the test dataset is $0.9942 \pm 0.00177$. In Fig. 5(a), the height of the peak is underestimated by about $10 \%$, while the slice energy spread is overestimated by less than $20 \%$. In Fig. 5(b), the height of the peak is underestimated by about $12 \%$, and the slice energy spread is only slightly overestimated at the center of the bunch. It should be noted that the peak shown in Fig. 5(a) is twice as high as that shown in Fig. 5(b) due 

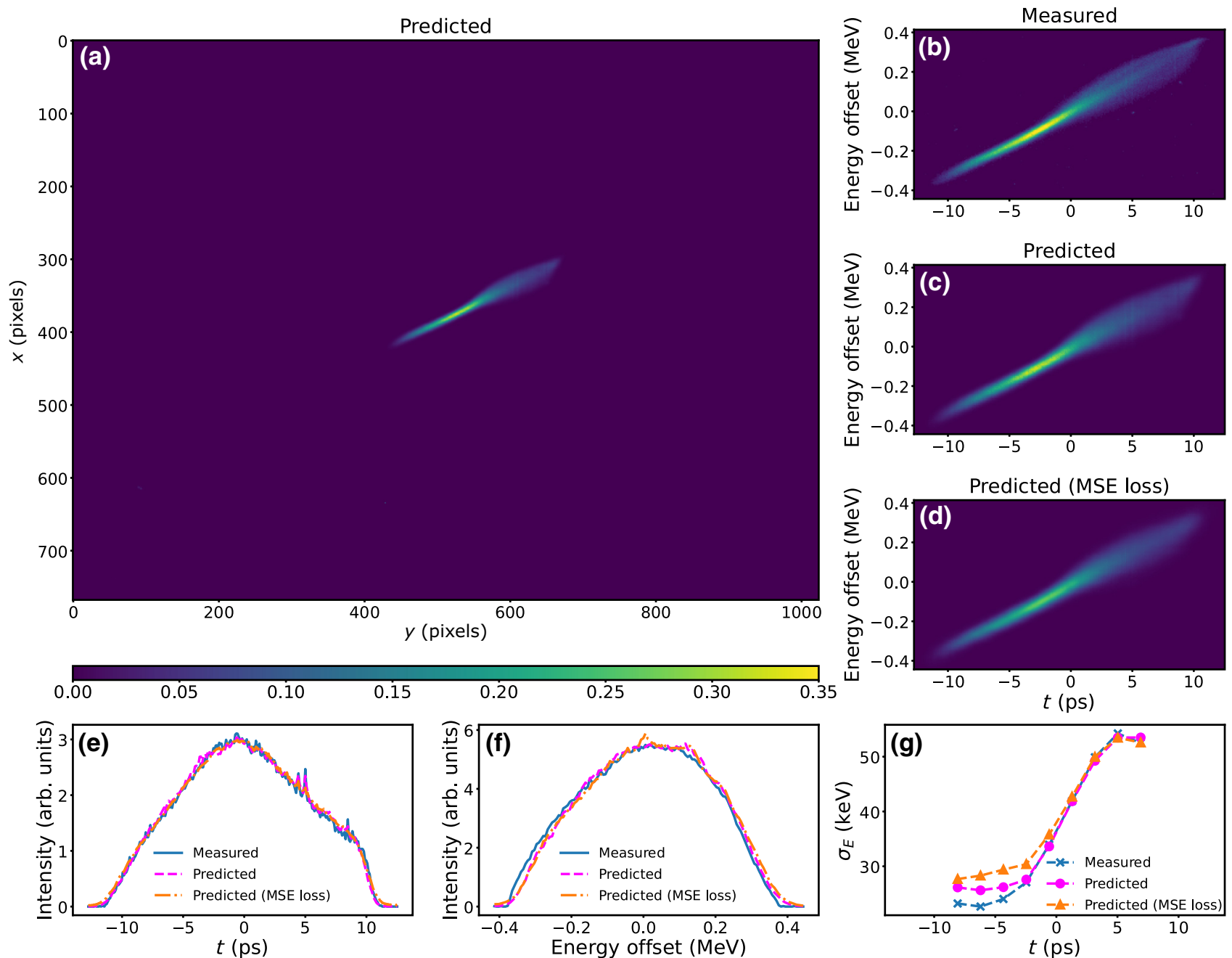

FIG. 4. (a) Example of an entire predicted image. The relative phases of the gun, A1, and AH1 are $-1.17^{\circ},-1.38^{\circ}$, and $0.04^{\circ}$, respectively. (b)-(d) Longitudinal phase spaces cropped from the measured image, the predicted image, and the image predicted by the model using the MSE as the loss function, respectively. (e)-(g) Comparisons of the current profiles, the energy spectra, and the rms slice energy spreads $\sigma_{E}$ between the longitudinal phase spaces shown in (b)-(d).

to the effect of AH1. As explained above, the precision of the model decreases as the number of pixels that represent the distribution decreases. Nevertheless, the prediction and the measurement agree well even in these extreme cases. Consequently, it can be inferred that the model is able to predict longitudinal phase spaces with high peak currents in a scenario where a parameter change results in a dramatic change in the current profile while the energy spectrum is stable.

\section{Irregular phase space}

Despite the strong nonlinearities during the emission and transport processes of electron bunches, the shapes of the longitudinal phase spaces in WP1 and WP2 are considered to be regular compared with those after electron bunches have been compressed strongly [29] or undergone energy modulation [30]. In order to verify that the proposed model is applicable to phase spaces with irregular shapes, the measured images for WP1 are distorted using the following equation:

$$
\mathbf{y}[i, j]=\mathbf{y}[i, j+40 \sin (j \pi / 45)],
$$

where $i$ and $j$ are the row and column indices, respectively, of the image data. The model for WP1 is trained from scratch on data created with the distorted images using the same procedure and hyperparameters as before. The resulting prediction and the distorted measurement still agree excellently, as shown in Fig. 6. The performance of the model over the test dataset is $0.9961 \pm 0.00199$. Although the distortion of the phase space is not induced by nonlinear beam dynamics, it indeed reflects the generality of the neural-network model. 
(a)

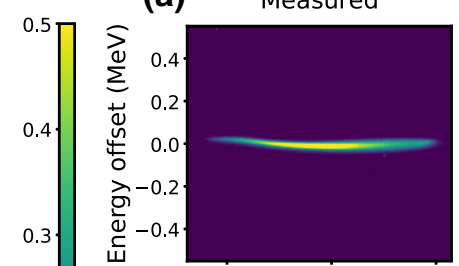

(b)

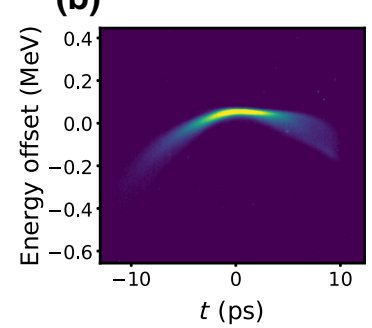

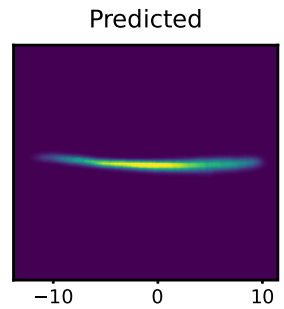

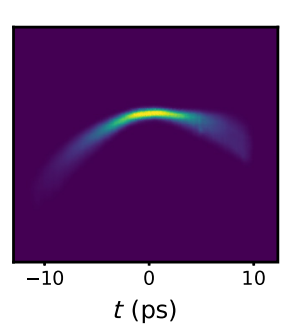

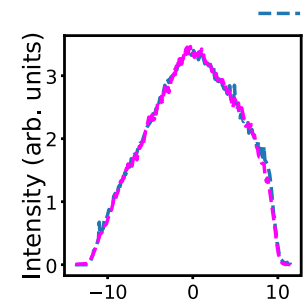
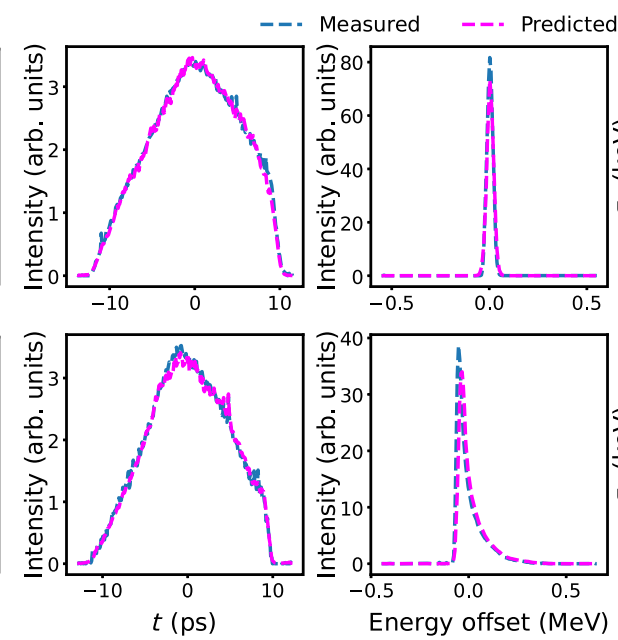
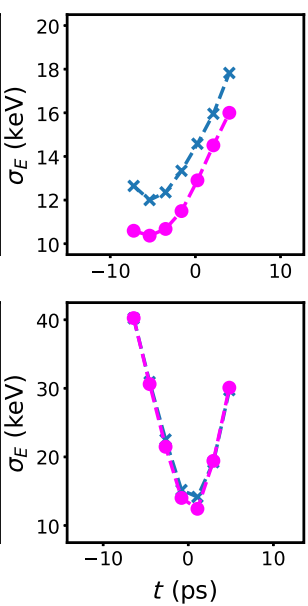

FIG. 5. Comparisons of the measured and predicted longitudinal phase spaces, current profiles, energy spectra, and rms slice energy spreads $\sigma_{E}$ for two shots with high peaks in the energy spectra. (a) The relative phases of the gun, $\mathrm{A} 1$, and $\mathrm{AH} 1$ are $-0.59^{\circ},-0.33^{\circ}$, and $-2.76^{\circ}$, respectively. (b) The relative phases of the gun and A1 are $-2.60^{\circ}$ and $0.20^{\circ}$, respectively. AH1 is switched off.

\section{More on the loss function}

As discussed previously, the coefficient $\alpha_{j}$ in Eq. (2) is critical to the performance of the model. We deliberately choose $\alpha_{j}<1$ to avoid overfitting on a single scale of the image. In other words, the model is not expected to generate a precise prediction, because the shot-to-shot jitter of machine parameters such as the arrival time of the photocathode laser is not available as input. To illustrate the outcome of overfitting, we train a model using the single-scale SSIM as the loss function. Namely, we ask the model to learn an exact mapping between the phase vector and the image. A typical result is shown in Fig. 7. The predicted longitudinal phase space is indeed close to the measured longitudinal phase space, except that the distribution is twisted along the longitudinal axis. Moreover, the agreement between the predicted and the measured current profiles is also not as good as the result shown in Fig. 5(a).

The characteristics of the sliding window $\mathbf{p}$ also affect the performance of the model. The standard SSIM uses a Gaussian sliding window of size $11 \times 11$ pixels. It is found that the performance of the model when trained with the uniform sliding window is slightly better than when it is trained with a Gaussian sliding window in terms of the current profile and the energy spectra, although the latter case generates a smoother image.

\section{SCALABILITY AND INTERPRETABILITY}

The design of the model is aimed at clearly separating the functions of the encoder and the decoder. Ideally, the encoder takes the input and generates latent features that contain information about the phase space of the electron bunch. The decoder translates the latent features into the corresponding diagnostic signal, which is the image on the screen in this study. This design leads to a scalable and interpretable model for a complex system because of parameter sharing. On the one hand, it is desirable to use the same latent features as the input for more decoders that model various diagnostics. This is also known as multitask learning [31]. On the other hand, different encoders can share a common decoder, as illustrated in Fig. 8(a), allowing multiple distinct working points to be integrated
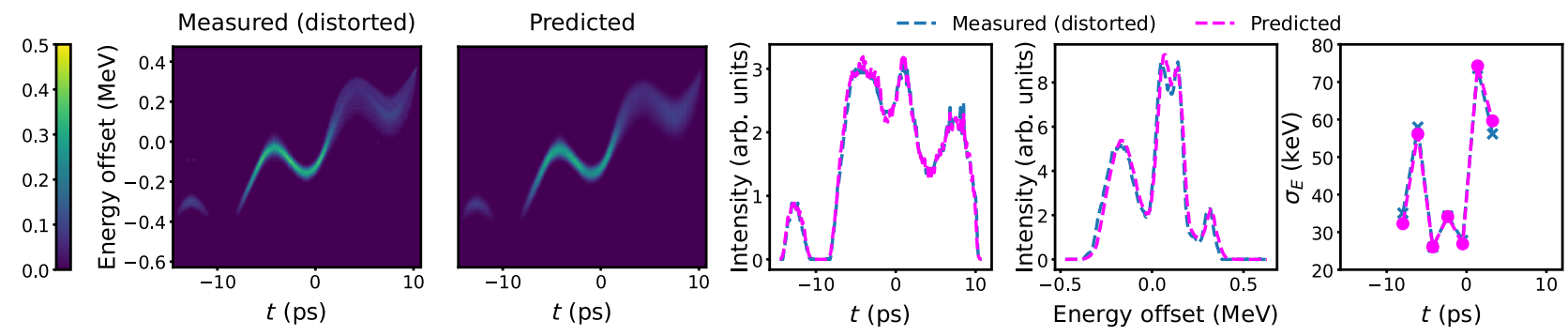

FIG. 6. Comparisons of corresponding measured (after distortion) and predicted longitudinal phase spaces, current profiles, energy spectra, and rms slice energy spreads $\sigma_{E}$. 

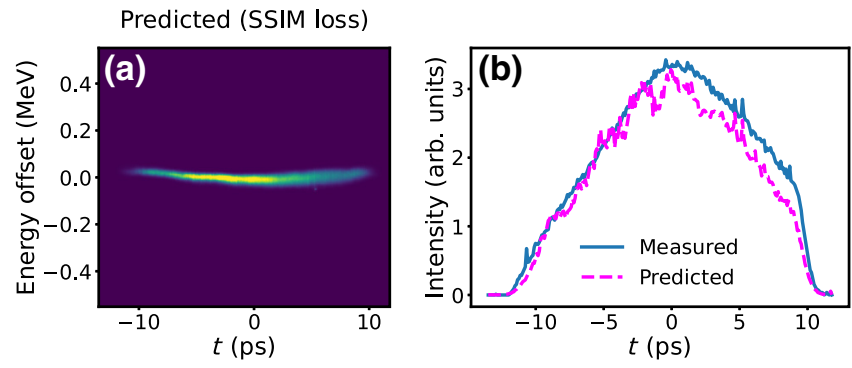

FIG. 7. (a) Prediction of the shot shown in Fig. 5(a) by the model using the SSIM as the loss function. (b) Comparison of current profiles between the predicted longitudinal phase space shown in (a) and the measured longitudinal phase space shown in Fig. 5(a).

into a single model. Separating the encoders for different working points is also practically necessary, because the time interval between the data collections for two working points can be significantly long, so that machine parameters not used as input may change due to long-term phenomena such as drift.

To prove the concept of the design, we utilize the decoder trained only for the WP1 branch to train the encoder for the WP2 branch from scratch. The weights in the decoder are frozen during training. Namely, only the encoder is trained. An example of the predicted longitudinal phase space is shown in Fig. 8(b). Although the decoder has not experienced any data without AH1 before, the predicted longitudinal phase space shows qualitative agreement with the measurement. The performance of the model over the test dataset is $0.9935 \pm 0.00165$. In order to improve the performance of the WP2 branch while preserving the performance of the WP1 branch, the model is further fine-tuned via the following steps:

(1) The branches for WP1 and WP2 are trained alternately for 100 epochs.

(2) The shared decoder is frozen while the encoders for WP1 and WP2 are trained for 100 epochs.

The above steps are repeated twice with two different learning rates: $3 \times 10^{-4}$ and $1 \times 10^{-4}$. As a result, the performance of the WP2 branch is significantly improved, as shown in Figs. 8(c)-8(e). The performance of the model over the test dataset increases to $0.9943 \pm 0.00177$ for WP2 and is $0.9956 \pm 0.00202$ for WP1, which are both as high as the corresponding metric achieved using the model without sharing the decoder. It should be noted that we do not optimize the above fine-tuning process, and thus it could take fewer epochs to reach the same performance. In the long run, it is expected that the decoder will become representative enough after it has been trained on enough (a)
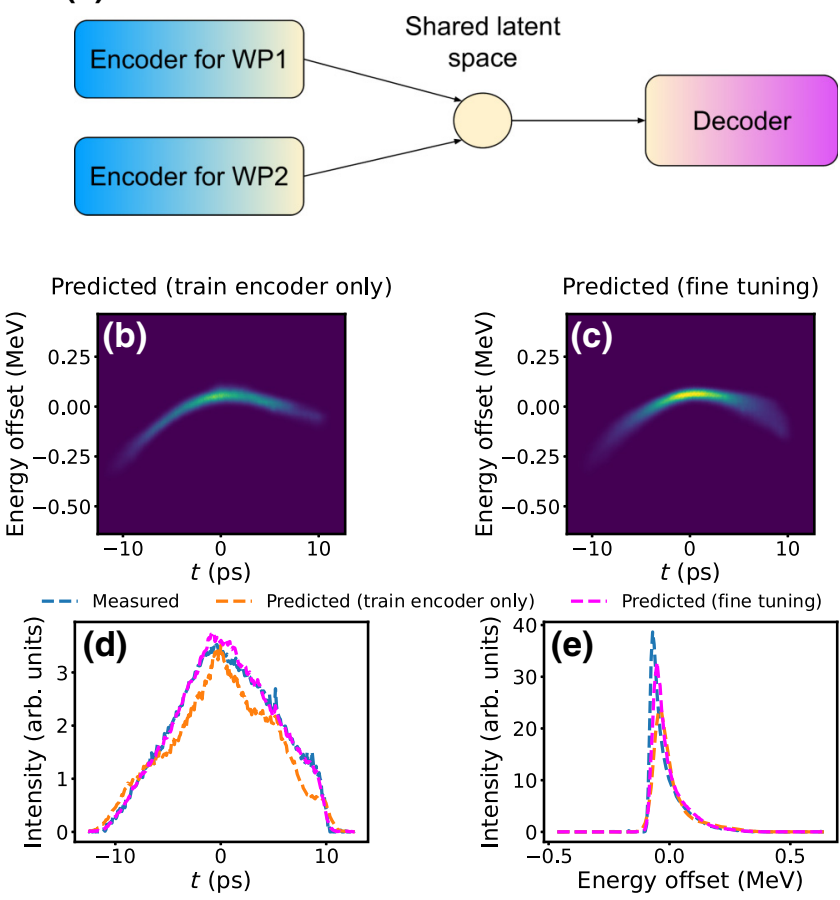

FIG. 8. Two different encoders share a common decoder. The measured longitudinal phase space for the example shot is shown in Fig. 5(b). (a) General architecture of the model. (b) Prediction of a shot for WP2 after the encoder is trained together with a frozen decoder trained only on WP1 data. (c) Prediction after the decoder and both the encoders are fine-tuned. (d),(e) Comparisons of current profiles and the energy spectra between the predicted longitudinal phase spaces in (b),(c) and the measurement.

data. Consequently, when a new working point is introduced, it will be necessary to train only a new encoder instead of the whole model with all the existing data.

Separating encoders for different working points allows one to build a large model incrementally. It also helps to reduce the dimension of the input data. For example, different AH1 gradients are set for WP1 and WP2, but the AH1 gradient does not need to be included in the input. Nevertheless, a large number of varying parameters could be needed for certain applications for a larger subsystem or a whole facility. Deep-learning technologies have been demonstrated to be able to solve extremely complex problems in an autonomous system provided that enough high-quality data and computational resources are available [32]. For scientific user facilities, the data-collection speed is determined by several factors, such as the repetition rate of the facility, the network delay, and the relaxation time when a parameter is changed, as well as the performance of the data acquisition system. If the influence of long-term phenomena (e.g., drift) in the facility is negligible, the methodology of lifelong learning [33] 
can be applied. Here, lifelong learning refers to the ability to continually learn over time by accommodating new knowledge while retaining previously learned experience. For example, data can be collected from routine operation and tuning, and then be filtered and cleaned for model training and fine tuning. Nonetheless, considerable effort is required to build such an intelligent and robust data pipeline.

\section{CONCLUSION}

In summary, we demonstrate modeling of the longitudinal phase-space-diagnostic beamline at the injector of the European XFEL using encoder-decoder neural-network models. After a model has been trained with experimental data only, it is capable of making high-fidelity predictions of megapixel images used for longitudinal phase-space measurement with $\mathrm{rf}$ phases as input. The predictions significantly outperform existing methods and are orders of magnitude faster than standard beam-dynamics simulations. The longitudinal phase space extracted from the predicted images agrees very well with the measurements not only visually, but also with respect to important physical properties such as the current profile, the energy spectrum, and the rms slice energy spread. Because of the constraints of the computational resources, the original images are downsampled by a factor of 2 . This downsampling can be avoided by utilizing a state-of-the-art graphics card or a distributed training strategy. Thus, fullsized camera images can be used to train the model without losing any information. In addition, a pragmatic way has been proposed to model a facility with various diagnostics and working points using deep neural networks. We show that the model is scalable and interpretable by sharing the same decoder with encoders used for different setups of the photoinjector. Moreover, the influence of the loss function that drives the training of the model is discussed in depth. We conclude that the impact of machine jitter can be mitigated by choosing proper values of the hyperparameters in the loss function, at the cost of some increase in blurring and loss of accuracy. On the other hand, the values of the hyperparameters should be adapted to improve the accuracy of the prediction if the machine jitter is negligible.

Because both the model and the loss function do not depend on any of the characteristics of an rf photoinjector or the longitudinal phase space of an electron bunch, we expect that this method can be generalized to many other image-based diagnostics, not only for accelerators but also for other types of scientific facility. The generality of the model is demonstrated by training the model on a dataset with artificially distorted longitudinal phase spaces. Looking forward, the model can be extended to include more diagnostics (decoders) for the longitudinal phase space and also for the transverse phase space, with the ultimate goal of building a complete virtual photoinjector using experimental data.

[1] W. Decking, S. Abeghyan, P. Abramian, A. Abramsky, A. Aguirre, C. Albrecht, P. Alou, M. Altarelli, P. Altmann, and K. Amyan et al., A MHz-repetition-rate hard $x$-ray free-electron laser driven by a superconducting linear accelerator, Nat. Photonics 14, 391 (2020).

[2] S. Pascarelli, S. Molodtsov, and T. Tschentscher, Creating a diverse international user facility, Nat. Rev. Phys. 2, 337 (2020).

[3] A. Sanchez-Gonzalez, P. Micaelli, C. Olivier, T. R. Barillot, M. Ilchen, A. A. Lutman, A. Marinelli, T. Maxwell, A. Achner, and M. Agåker et al., Accurate prediction of $x$-ray pulse properties from a free-electron laser using machine learning, Nat. Commun. 8, 15461 (2017).

[4] C. Emma, A. Edelen, M. J. Hogan, B. O'Shea, G. White, and V. Yakimenko, Machine learning-based longitudinal phase space prediction of particle accelerators, Phys. Rev. Accel. Beams 21, 112802 (2018).

[5] A. Edelen, N. Neveu, M. Frey, Y. Huber, C. Mayes, and A. Adelmann, Machine learning for orders of magnitude speedup in multiobjective optimization of particle accelerator systems, Phys. Rev. Accel. Beams 23, 044601 (2020).

[6] X. Ren, A. Edelen, A. Lutman, G. Marcus, T. Maxwell, and D. Ratner, Temporal power reconstruction for an $\mathrm{x}-$ ray free-electron laser using convolutional neural networks, Phys. Rev. Accel. Beams 23, 040701 (2020).

[7] X. Xu, Y. Zhou, and Y. Leng, Machine learning based image processing technology application in bunch longitudinal phase information extraction, Phys. Rev. Accel. Beams 23, 032805 (2020).

[8] C. Tennant, A. Carpenter, T. Powers, A. Shabalina Solopova, L. Vidyaratne, and K. Iftekharuddin, Superconducting radio-frequency cavity fault classification using machine learning at jefferson laboratory, Phys. Rev. Accel. Beams 23, 114601 (2020).

[9] S. Nagaitsev, Z. Huang, J. Power, J.-L. Vay, P. Piot, L. Spentzouris, J. Rosenzweig, Y. Cai, S. Cousineau, and M. Conde et al., Accelerator and beam physics research goals and opportunities, arXiv:2101.04107.

[10] A. Scheinker, A. Edelen, D. Bohler, C. Emma, and A. Lutman, Demonstration of Model-Independent Control of the Longitudinal Phase Space of Electron Beams in the Linac-Coherent Light Source with Femtosecond Resolution, Phys. Rev. Lett. 121, 044801 (2018).

[11] S. C. Leemann, S. Liu, A. Hexemer, M. A. Marcus, C. N. Melton, H. Nishimura, and C. Sun, Demonstration of Machine Learning-Based Model-Independent Stabilization of Source Properties in Synchrotron Light Sources, Phys. Rev. Lett. 123, 194801 (2019).

[12] C. Emma, A. Edelen, A. Hanuk, B. O'Shea, and A. Scheinker, Virtual diagnostic suite for electron beam prediction and control at facet-ii, Information 12, 61 (2021).

[13] I. Goodfellow, Y. Bengio, and A. Courville, Deep Learning (MIT Press, Cambridge, MA, 2016), http://www.deeplearn ingbook.org. 
[14] N. A. Moody, K. L. Jensen, A. Shabaev, S. G. Lambrakos, J. Smedley, D. Finkenstadt, J. M. Pietryga, P. M. Anisimov, V. Pavlenko, and E. R. Batista et al., Perspectives on Designer Photocathodes for $x$-Ray Free-Electron Lasers:influencing Emission Properties with Heterostructures and Nanoengineeredelectronic States, Phys. Rev. Appl. 10, 047002 (2018).

[15] Y. Chen, I. Zagorodnov, and M. Dohlus, Beam dynamics of realistic bunches at the injector section of the european $x$-ray free-electron laser, Phys. Rev. Accel. Beams 23, 044201 (2020).

[16] J. Qiang, Y. Ding, P. Emma, Z. Huang, D. Ratner, T. O. Raubenheimer, M. Venturini, and F. Zhou, Start-to-end simulation of the shot-noise driven microbunching instability experiment at the linac coherent light source, Phys. Rev. Accel. Beams 20, 054402 (2017).

[17] A. Edelen, N. Neveu, C. Emma, D. Ratner, and C. Mayes, in NeurIPS Machine Learning for the Physical Sciences Workshop (NeurlPS2019, Vancouver, Canada, 2019).

[18] D. P. Kingma and M. Welling, An introduction to variational autoencoders, Found. Trends Mach. Learn. 12, 307 (2019).

[19] I. J. Goodfellow, J. Pouget-Abadie, M. Mirza, B. Xu, D. Warde-Farley, S. Ozair, A. Courville, and Y. Bengio, Generative adversarial networks, arXiv:1406.2661.

[20] V. Dumoulin and F. Visin, A guide to convolution arithmetic for deep learning, arXiv:1603.07285v2.

[21] C. Ledig, L. Theis, F. Huszár, J. Caballero, A. Cunningham, A. Acosta, A. Aitken, A. Tejani, J. Totz, Z. Wang, and W. Shi, in 2017 IEEE Conference on Computer Vision and Pattern Recognition (CVPR) (IEEE, Honolulu, HI, USA, 2017), p. 105.

[22] Z. Wang, A. Bovik, H. Sheikh, and E. Simoncelli, Image quality assessment: From error visibility to structural similarity, IEEE Trans. Image Process. 13, 600 (2004).

[23] F. Brinker, in 7th International Particle Accelerator Conference (JACoW, Busan, Korea, 2016), p. TUOCA03.
[24] R. Akre, L. Bentson, P. Emma, and P. Krejcik, in PACS2001. Proceedings of the 2001 Particle Accelerator Conference (Cat. No.01CH37268) (IEEE, Chicago, IL, USA, 2001), Vol. 3, p. 2353.

[25] M. Abadi, A. Agarwal, P. Barham, E. Brevdo, Z. Chen, C. Citro, G. S. Corrado, A. Davis, J. Dean, and M. Devin et al., Tensorflow: Large-scale machine learning on heterogeneous distributed systems, arXiv:1603.04467v2, software available from tensorflow.org.

[26] K. He, X. Zhang, S. Ren, and J. Sun, in 2015 IEEE International Conference on Computer Vision (ICCV) (IEEE, Santiago, Chile, 2015), p. 1026.

[27] Diederik P. Kingma and J. Ba, Adam: A method for stochastic optimization, arXiv:1412.6980.

[28] L. Winkelmann, A. Choudhuri, H. Chu, I. Hartl, C. Li, C. Mohr, J. Mueller, F. Peters, S. Pfeiffer, S. Salman, and U. Grosse-Wortmann, in 39th Free Electron Laser Conference (JACoW, Hamburg, Germany, 2019), p. WEP046.

[29] J. Zhu, R. W. Assmann, M. Dohlus, U. Dorda, and B. Marchetti, Sub-fs electron bunch generation with sub10-fs bunch arrival-time jitter via bunch slicing in a magnetic chicane, Phys. Rev. Accel. Beams 19, 054401 (2016).

[30] S. Antipov, C. Jing, M. Fedurin, W. Gai, A. Kanareykin, K. Kusche, P. Schoessow, V. Yakimenko, and A. Zholents, Experimental Observation of Energy Modulation in Electron Beams Passing through Terahertz Dielectric Wakefield Structures, Phys. Rev. Lett. 108, 144801 (2012).

[31] M. Crawshaw, Multi-task learning with deep neural networks: A survey, arXiv:2009.09796.

[32] S. Grigorescu, B. Trasnea, T. Cocias, and G. Macesanu, A survey of deep learning techniques for autonomous driving, J. Field Robot. 37, 362 (2020).

[33] G. I. Parisi, R. Kemker, J. L. Part, C. Kanan, and S. Wermter, Continual lifelong learning with neural networks: A review, Neural Netw. 113, 54 (2019). 